\title{
Clinical feasibility of deep hyperthermia treatment in the head and neck; new challenges for positioning and temperature measurement.
}

\author{
M.M. Paulides ${ }^{1}$, J.F. Bakker ${ }^{1}$, M. Linthorst ${ }^{1}$, J. van der \\ ${ }^{1}$ Department of Radiation Oncology, Erasmus MC - Daniel den Hoed Cancer \\ Center, Rotterdam, The Netherlands. \\ ${ }^{2}$ Foundation for Research on Information Technologies in Society (IT'IS), \\ Zurich, Switzerland. \\ ${ }^{3}$ Department of Radiology, Erasmus MC, Rotterdam, The Netherlands. \\ E-mail: m.paulides@erasmusmc.nl
}

\begin{abstract}
To apply high quality hyperthermia treatment to tumours at deep locations in the head and neck $(\mathrm{H} \& \mathrm{~N})$ we have designed and built a site-specific phased-array applicator. Earlier, we demonstrated its features in parameter studies, validated those by phantom measurements and clinically introduced the system. In this publication we will critically review our first clinical experiences and demonstrate the pivotal role of hyperthermia treatment planning (HTP).

Three representative patient cases (thyroid, oropharynx and nasal cavity) are selected and discussed. Treatment planning, the treatment, interstitially measured temperatures and their interrelation are analyzed from a physics point of view.

Treatments lasting one hour were feasible and well tolerated and no acute treatment-related toxicity has been observed. Maximum temperatures measured are in the range of those obtained during deep hyperthermia treatments in the pelvic region but mean temperatures are still to be improved. Further, we found that simulated power absorption correlated well with measured temperatures illustrating the validity of our treatment approach of using energy profile optimizations to arrive at higher temperatures.

This is the first data proving that focussed heating of tumours in the H\&N is feasible. Further, HTP proved a valuable tool in treatment optimization. Items to improve are (1) the transfer of HTP settings into the clinic and (2) the registration of the thermal dose, i.e. dosimetry.
\end{abstract}

PACS numbers: 41,87.50.st,87.55.D-,87.55 


\section{Introduction}

Treatment of advanced tumours in the H\&N region remains complex (Conley 2006) and loco-regional control still poses a major therapeutic challenge (El-Sayed \& Nelson 1996, Pignon et al. 2000). The currently standard radio-chemotherapy (RCT) 5 generally results in a 5-year survival rate of $20 \%$ to $65 \%$ (Conley 2006). The metaanalysis from Bourhis et al. (Bourhis et al. 2006) showed that the highest benefit can be expected from hyper-fractionated RT without total dose reduction but that further improvement is warranted since loco-regional recurrence rates are up to 30$40 \%$. Also, the enhanced disease control carries a price tag since the burden of toxicity 10 in this group of patients is severe (El-Sayed \& Nelson 1996, Cummings et al. 2007). This toxicity incidence shows a sharp increase with RT-dose, e.g. swallowing complaints increase by $18 \%$ per 10 Gy beyond a mean dose of 55 Gy (Levendag et al. 2007) and saliva production is strongly reduced for RT-doses above the threshold value (Jensen et al. 2007, Jellema 2007, Terhaard 2008). Given the demonstrated 15 ability of hyperthermia (HT) to enhance treatment outcome when added to (hyperfractionated) RT, without increasing toxicity (Amichetti et al. 1997, Franckena et al. 2009, Kampinga 2006, van der Zee et al. 2008, Sugahara et al. 2008), it seems logical to combine RT with HT to further improve the therapeutic ratio in the treatment of $\mathrm{H} \& \mathrm{~N}$ cancer. This is even more accentuated as modern HT

20 offers exciting opportunities to target electromagnetic energy to the tumor (Paulides, Bakker, Neufeld, Van der Zee, Jansen, Levendag \& Van Rhoon 2007). Adding targeted HT to targeted RT potentially results in a substantially increased biological equivalent tumor RT dose, while at the same time the RT-dose in the areas at risk might be reduced to below the threshold level.

Until recently, the application of HT in the H\&N region was limited to superficial regions, mostly lymphnodes, because of the lack of deep-heating equipment for this region. The development of the HYPERcollar applicator system has bridged this gap by providing the technology to apply deep heating in the H\&N region (Paulides, Bakker, Neufeld, Van der Zee, Jansen, Levendag \& Van Rhoon 2007, Bakker

30 et al. n.d.). A phased-array of twelve antennas and the use of a high frequency, i.e. $433 \mathrm{MHz}$, features tailoring the specific absorption rate (SAR) pattern for maximum heating in the target region while minimizing the heat in critical tissues (i.e. spinal column) (Paulides et al. 2005, Paulides, Bakker, Zwamborn \& Van Rhoon 2007). Highly detailed patient-specific HTP is exploited for maximum energy focussing

(Neufeld 2008). Ideally, in HTP one would optimize the temperature pattern. However, this pattern depends on several thermal parameters that vary in time, in patients and between patients. Hence, usually SAR-based HTP is used to obtain optimum phase and power settings. Phantom measurements have experimentally shown simulation accuracy (Paulides, Bakker \& Van Rhoon 2007) but a clinical

40 validation is difficult: focussed heating results in steep SAR gradients (Paulides et al. 2005, Paulides, Bakker, Zwamborn \& Van Rhoon 2007) and these gradients, combined with the presence of large vessels and air, convert into large gradients in the thermal distribution. Hence, the clinical validation is a challenging task that should be rigorously approached.

45 In this publication, we evaluate the application of HT using the HyperCollar applicator system (Paulides, Bakker, Neufeld, Van der Zee, Jansen, Levendag \& Van Rhoon 2007). Worldwide the first data on deep heating in the H\&N using a phased-array approach is presented for three sub-locations, i.e. thyroid (larynx), 
oropharynx and nasal cavity (nasopharynx). Measured temperatures and simulated SAR values are compared to verify our approach of improving temperatures by exploiting SAR-based treatment planning. Overall, the objective of this paper is to provide a critical evaluation of the possibilities, limitations and challenges of the

5 technology used and pave the way for a future clinical validation study.

\section{Methods and materials}

From the H\&N patients treated with hyperthermia, we selected three representative patient cases. Details on (1) treatment planning, (2) the clinical application of the treatment, (3) the temperatures and (4) acute effects are reported. As this is not a 10 study to assess the clinical outcome, we will only provide the information required to analyze the quality of the treatment applied from a technological point of view. The results of this evaluation form the input to discuss requirements to further improve treatment quality.

\section{Treatment planning}

15 Ideally, in HTP one would optimize the temperature pattern. However, temperature profiles in human tissue during hyperthermia depend on the combined influences of applied power and several cooling mechanisms. These mechanisms are difficult to predict since cooling depends heavily on thermal parameters that vary in time, in patients and between patients. Therefore, we limited HTP to SAR predictions

20 and assumed that a high SAR level will correlate with a high temperature. Hence, to find the optimum treatment settings (patient positioning and power and phase of the signals), we performed SAR-based HTP, for all patients, using SEMCAD $\mathrm{X}$ (Neufeld 2008). HTP is started by acquiring a $\mathrm{H} \& \mathrm{~N}$ radiotherapy-planning computerized tomography (CT) scan ("planning CT"), with contrast to enhance tumour delineation. With the CT, the medical doctor (MD) constructs a patient model ("segmentation") in about six hours. This procedure is semi-automatic, i.e. discrimination between areas of exterior, fat, muscle and bone is automatic, with a manual fine-tuning, and essential "soft" tissues such as tumour, cartilage, white matter and gray matter are assigned manually. In a last step, couch, neck support and patientspecific RT mask have to be removed. The segmentation and a CAD implementation of the applicator are imported in SEMCAD X and tissue and material properties (as in (Paulides, Bakker, Neufeld, Van der Zee, Jansen, Levendag \& Van Rhoon 2007)) are assigned to these solid models. With this set-up, the electric fields for each antenna are calculated using the electromagnetic solver of SEMCAD X.

Amplitude (power) and phases of these fields are optimized by using the optimization procedure of Kohler et al. (Kohler et al. 2001) as described in (Neufeld 2008). As in (Paulides, Bakker, Neufeld, Van der Zee, Jansen, Levendag \& Van Rhoon 2007), we optimized the weighted SAR ratio in the target region versus the SAR in the non-target region. The weight of the total amount of SAR in a certain region (tissue or user-defined volume) can be selectively increased or decreased by adapting their respective weight factors. By choosing appropriate weight factors, it is possible to adjust the SAR patterns such that all target regions are sufficiently covered by the preferred iso-SAR contour (Paulides, Bakker, Neufeld, Van der Zee, Jansen, Levendag \& Van Rhoon 2007). This is still a manual iterative process since we have no tool yet to optimize iso-SAR coverage. However, using this procedure, additional 
target or sensitive regions can be used to maximize or minimize total SAR in those regions. This can be used to elegantly convert complaints of patients into sensitive regions where the local SAR can be minimized. The final aim is to exploit this option during treatment but, for this study, such complaint-based re-optimization was only

5 applied between treatments because the procedure was not yet fast enough to apply online.

SAR distributions (1 g averaged Spatial Peak SAR [IEEE-1529]) are visualized using SAR cross-sections, cumulative SAR-Volume histograms and iso-SAR volumes at the $25 \%$ of the maximum value level, as introduced and discussed in (Paulides,

10 Bakker, Neufeld, Van der Zee, Jansen, Levendag \& Van Rhoon 2007). Further, we define the tumour coverage (TC) as the percentage of the total tumour volume that is covered by at least $25 \%$ of these SAR distributions.

\section{Treatment procedure}

Radiotherapy was applied to the primary tumour and lymph node stations according to the standard radiation schedule for the respective subgroup and hyperthermia was added once or twice a week depending on the length of the radiation schedule.

Before heating, closed-tip thermometry catheters are placed interstitially, intraluminally and/or at the skin. Depending on feasibility and risk of toxicity, interstitial catheters are placed in the tumour and in muscle tissue with similar predicted SAR values as the spinal cord ("myelum indicative") under CT guidance or under anesthesia in an operating room. Intraluminal catheters are placed, through the nose, in the esophagus near a laryngeal or oropharyngeal tumour, or in a brachyradiotherapy nasopharynx applicator ("mould") (Levendag et al. 1997), which is positioned according to the procedure in (Kolkman-Deurloo 2007) (section 2.3.8).

25 After catheter placement, a specific CT scan ("thermometry CT") is made to accurately document the locations of the catheter tracts.

In the hyperthermia treatment room, the patient is placed on an ordinary patient bed. The tumour is placed centrally within the applicator, as close as permitted by the shoulders, by adjusting the patient position with respect to the applicator ring. so In this procedure, the height of head and shoulders are adjusted by changing the height of the neck support and patient-bed (Paulides, Bakker, Neufeld, Van der Zee, Jansen, Levendag \& Van Rhoon 2007) (Figure 1) in accordance to planned positions. After patient positioning, four-sensor optical thermometry fibers $\$$ are placed into the interstitial and intraluminal closed-tip catheters. Additional thermometry probes are 35 placed on the skin and at the inflow and outflow of the waterbolus. Subsequently, the waterbolus is filled with demineralized water that is circulated at a temperature in the range of $20-30^{\circ} \mathrm{C}$, depending on the depth of the tumour. If the target area extends to less than $1 \mathrm{~cm}$ from the skin, extra temperature controlled waterbolus bags are applied, at a temperature around $40^{\circ} \mathrm{C}$.

40 The heat session is started by applying $80 \mathrm{~W}$ of total power with the power and phase control system (Bakker et al. n.d.), using the optimized phase and power settings from HTP. Power is increased subsequently in steps of $30 \mathrm{~W}$, usually around one step per minute, till one of the tolerance limits is reached $\left(40^{\circ} \mathrm{C}\right.$ in myelum-indicative, $60 \mathrm{~W} / \mathrm{kg}$ in myelum predicted by $\mathrm{HTP}, 43^{\circ} \mathrm{C}$ in other tissues) or the occurrence of a

45 hot spot indicated by the patient at a site without thermometry. Two phases of a

$\ddagger$ Takaoka FTP1 standard sensor probes (Takaoka Electric MFG Co. Ltd., Japan) 

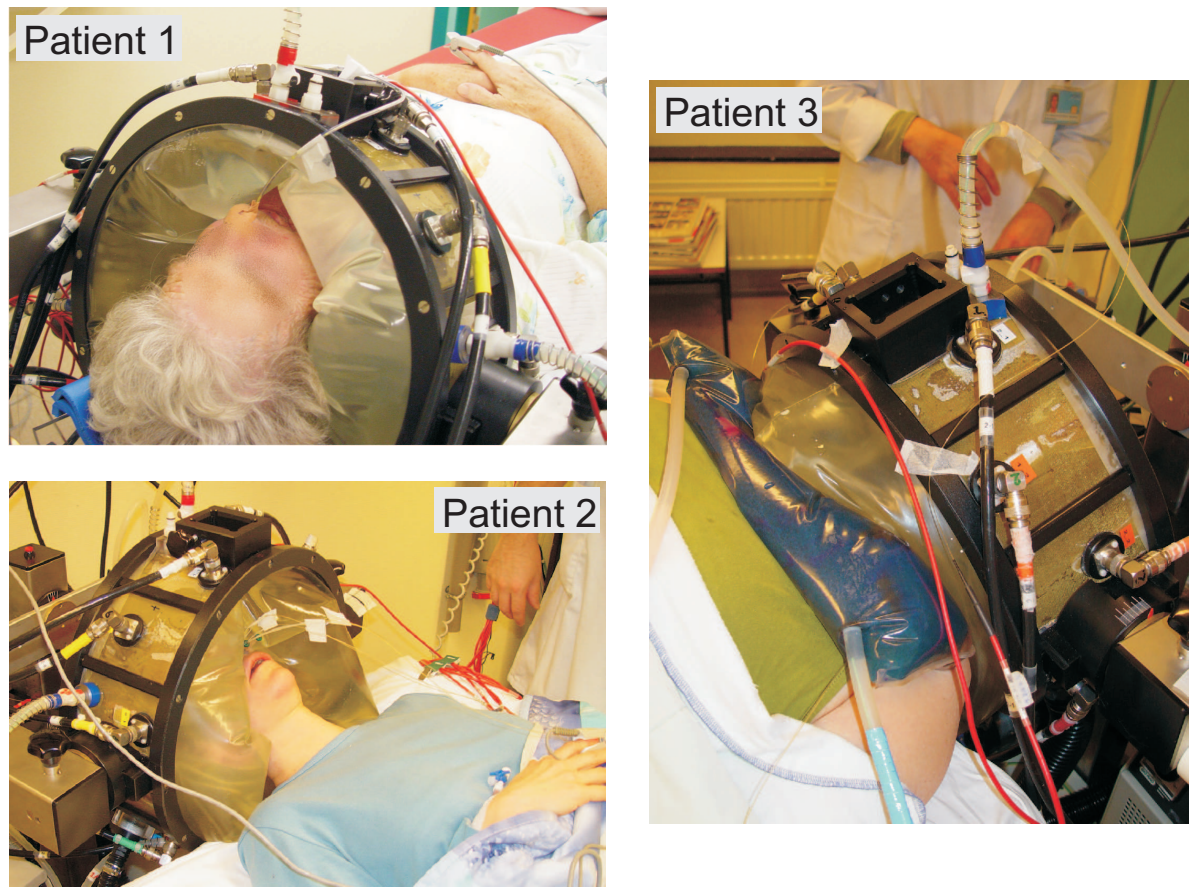

Figure 1. Pictures of the setups for the three patients discussed in this paper, with tumours in 1) oropharynx, 2) nasopharynx/nasal cavity and 3) thyroid respectively. Note the different arrangement of the setup to treat patient 2 and the additional waterbolus between patient 3 and the HYPERcollar.

treatment are defined for data analysis: (1) "warm-up phase", (2) "plateau-phase", and the transition is assumed to be always after 15 minutes of heating (Figure 6).

\section{Comparison of measurements to simulations}

To verify our approach of optimizing SAR by simulations to achieve higher 5 temperatures, we studied the correlation between measured temperatures and calculated local SAR values. Hereto we selected the temperature probes where we expected reliable results, i.e. we used only temperature measurements of probes at least $5 \mathrm{~mm}$ in tissue. Hence, intraluminal probe measurements were omitted and interstitial temperatures close to the skin surface or tissue surface, e.g. in the nostrils, are omitted due to large SAR uncertainties (FDTD staircasing and partial volume effects) and extreme influence of thermal conduction (waterbolus and nasal/oral cavities). The locations of the probes were precisely reconstructed by segmenting the catheter tracks in the thermometry CT and manually registering the thermometry CT to the planning CT. After treatment, the SAR distributions were recalculated using patient positioning, power and phase settings as measured during treatment. Subsequently, we determined the correlation between measured temperatures, at start and at time-points where the temperature was stable, and the corresponding recalculated local SAR values. 
Table 1. Patient, HTP and treatment statistics for the three patients. T-vol denotes the segmented tumour volume, TC is the predicted tumour coverage $\left(25 \%\right.$ iso-SAR $\left.\operatorname{Sax}_{\max }\right)$, \#HT denotes the number of heating sessions given. $\bar{P}_{\text {tot }}$, $\bar{T}_{W B}, \bar{T}_{T}$ denote the range of mean values during plateau phase (average in both time and \#HT) of the total applied power, the waterbolus temperature and the temperature measured interstitially in the tumour, respectively, and $\bar{T}_{T, \max }$ the range of maximum measured temperatures.

\begin{tabular}{lccccccc}
\hline Patient & $\begin{array}{c}\text { T-vol } \\
\left(\mathrm{cm}^{3}\right)\end{array}$ & TC & \#HT & $\begin{array}{c}\bar{P}_{t o t} \\
(\mathrm{~W})\end{array}$ & $\begin{array}{c}\bar{T}_{W B} \\
\left({ }^{\circ} \mathrm{C}\right)\end{array}$ & $\begin{array}{c}\bar{T}_{T} \\
\left({ }^{\circ} \mathrm{C}\right)\end{array}$ & $\begin{array}{c}\bar{T}_{T, \max } \\
\left({ }^{\circ} \mathrm{C}\right)\end{array}$ \\
\hline 1. Oropharynx & 27 & $85 \%$ & 5 & 434 & 26 & $37.7-40.8$ & $38.2-42.0$ \\
2. Nasal cavity & 40 & $58 \%$ & 3 & 438 & 22 & $38.9-39.5$ & $39.7-40.1$ \\
3. Thyroid & 114 & $94 \%$ & 4 & 346 & 26 & $36.9-40.7$ & $37.1-42.9$ \\
& & & & & 39.4 & & \\
\hline
\end{tabular}

\section{Selection of cases}

From the group of patients treated with the HYPERcollar, every first patient per sublocation ("larynx/thyroid", "oropharynx" and "nasopharynx/nasal cavity") for whom interstitial thermometry was available was selected (Figure 11). In this way,

5 patient selection was not biased by the treatment team since the feasibility of interstitial thermometry was decided upon by an experienced MD (e.g. a radiologist) for each individual patient.

\section{Results}

\section{Patient 1: Oropharynx}

10 This 82-year-old female was treated for a recurrent tumour in the base of the tongue and a lymphnode metestasis. She had been treated 26 years before with chemotherapy, surgery and radiotherapy for a T1N1M0 carcinoma of the tonsillar region. She refused surgery of the base of the tongue tumour and treatment comprised radiotherapy (IMRT: 20 x 2.5Gy, 5/wk) combined with four heating sessions (1/wk). Three 15 catheters were placed interstitially in the tumour using the procedure that is normally used for interstitial (brachy) radiotherapy treatment in the base of tongue ((KolkmanDeurloo 2007), section 2.3.2). Prior to HT treatment, an intraluminal catheter was placed through the nose in the esophagus such that two thermometry-probes could be positioned near the tumour. We identified 3 metallic pins (diameter $\sim 5 \mathrm{~mm}$, length $\sim 10 \mathrm{~mm}$ ) in the jaws as possibly treatment-limiting but HTP disproved our initial hesitation.

Treatment planning revealed a TC of $85 \%$ but also a severe hotspot at the left shoulder. During the first heating session we used a maximum of $570 \mathrm{~W}$ with temperatures up to $42.0^{\circ} \mathrm{C}$ (Figure 7) but temperatures at the back of the tumour remained low (maximum $\sim 39^{\circ} \mathrm{C}$, mean $\sim 38^{\circ} \mathrm{C}$ ), probably due to low SAR levels as already predicted by HTP. Also consistent with HTP, the treatment was hampered by a persisting hotspot at the left shoulder. Therefore, after the first treatment, we performed an additional optimization cycle, using the shoulders as sensitive regions where the SAR level should be minimized. The re-optimized settings resulted in a higher tolerated maximum power level in the remaining treatments $(650 \mathrm{~W}-850 \mathrm{~W})$ and mean measured temperatures showed an initial increase of $\sim 1^{\circ} \mathrm{C}$. Further, in 


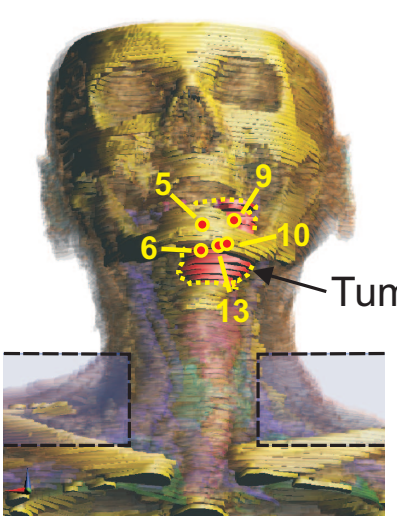

Anatomy

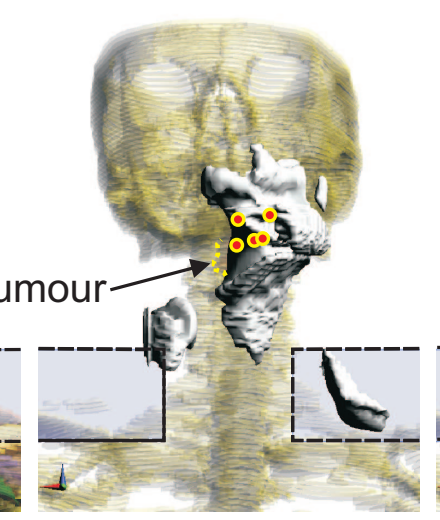

Original

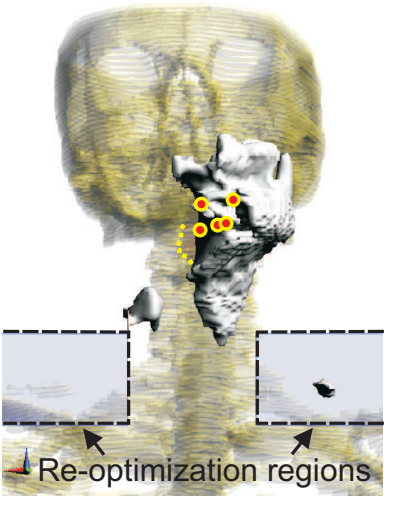

Re-optimized

Figure 2. [Patient 1: Oropharynx] Frontal views of the anatomy, with measurement probe locations ("anatomy") and $25 \%$ iso-SAR distributions (in grey) predicted by SEMCAD X for the first ("original") optimized settings and for the "re-optimized" settings. The projected locations of the measurement locations are illustrated by the yellow circles filled with red. Note that the SAR in the reoptimization region (left shoulder) is effectively reduced in this step and also the SAR at the right side of the neck is decreased.

accordance with HTP, no complaints at the shoulders occured (Figure 21). Figure 7 shows the temperatures obtained during the four heating sessions. This figure shows that, for patient 1 (but also patient 2), the temperatures measured in interstitial catheters are on average higher than those measured intraluminally.

Although the intra-tumour temperatures varied between treatments, results indicate that therapeutic temperatures can be obtained (Fatehi, van der Zee, de Bruijne, Franckena \& van Rhoon 2007, Franckena et al. 2009). Since this treatment was at the very start of the learning curve, i.e. less than 3 years as indicated for deep HT by Fatehi et al(Fatehi, van der Zee, de Bruijne, Franckena \& van Rhoon 2007), we believe that a combination of experience and enhancing the waterbolus shape suffices to correct for this variation in treatment quality. Overall, SAR hotspots predicted by HTP correlated very well with pain complaints of this patient and re-optimization proved an adequate way to reduce hotspots. Hence, this case illustrates the high potential benefit of online pain-based SAR steering, e.g. as in Canters et al (Canters et al. 2008).

\section{Patient 2: Nasal cavity}

This patient was a 38-year old female with a large adenoid cystous carcinoma of the nasopharynx and nasal cavity. She had been treated with radiotherapy for a meningeoma of the cavernous sinus to the same region 3 years earlier. Radiation with the Cyberknife (5x5.5Gy, in 9 days with 2 days spacing) was combined with 3 sessions of hyperthermia. Interstitial catheters were placed under anaesthesia in the front part of the tumour (Figure 3). Additionally, a mould was positioned ((KolkmanDeurloo 2007) section 2.3.8) for intraluminal measurements close to the tumour.

The HYPERcollar was positioned over the middle of the head, with the eyes covered with the waterbolus (Figure 1). HTP was performed with this setup and 

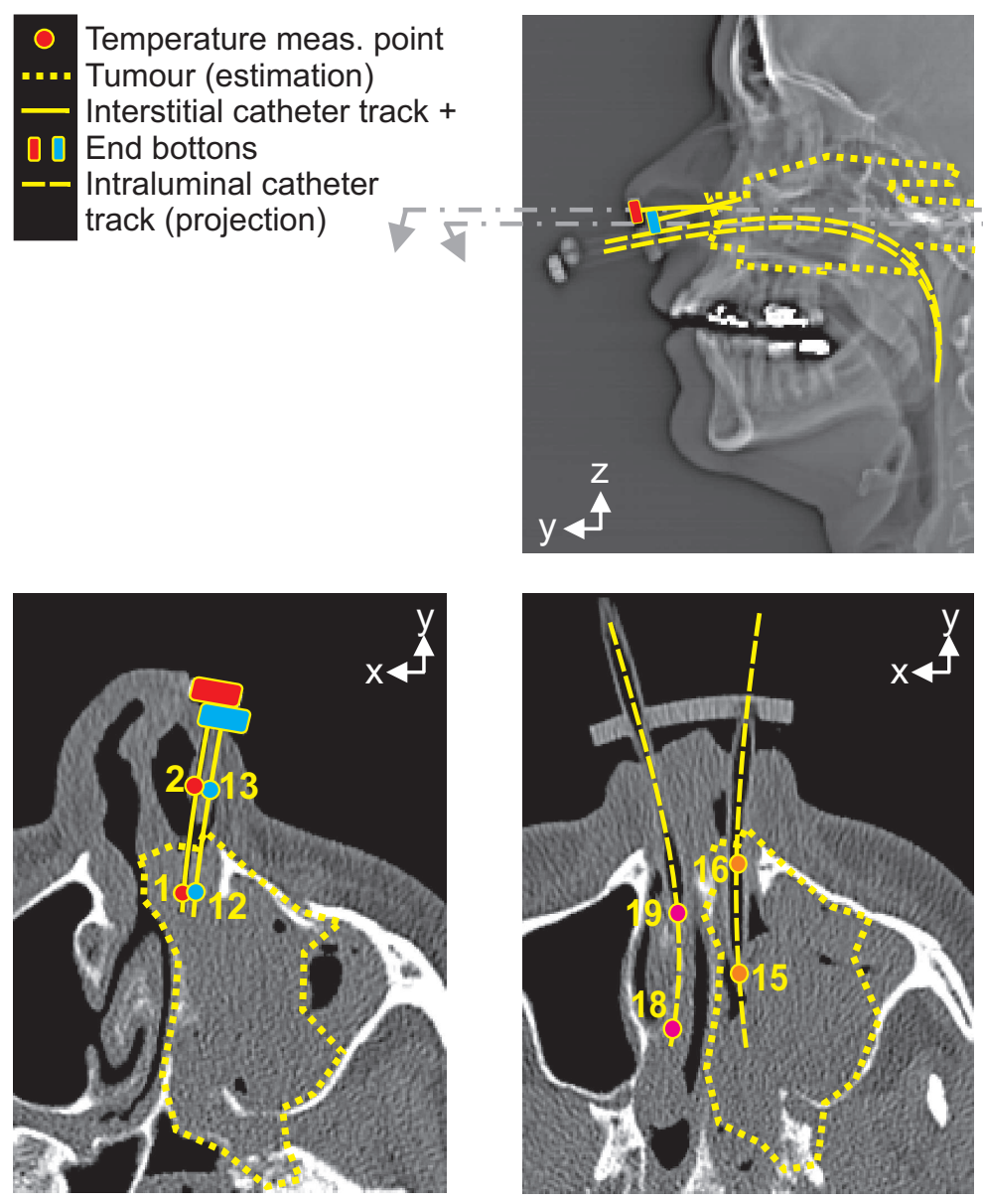

Figure 3. [Patient 2: Nasal cavity] CT-slices and overview x-ray (top-right) with indicated the real tracks of the interstitial (bottom-left) and a projections of the intraluminal (bottom-right) catheters and corresponding measurement locations. The catheter at point 16 is fully enclosed in tissue whereas points 15, 18 and 19 have only partial thermal tissue contact (limitedly visible in these views).

revealed a maximum TC of $58 \%$, but showed that the SAR levels in the eyes and myelum would remain low. Lowest SAR was predicted to be in the center of the tumour (Figure 4). Although the TC did not meet with our initial inclusion criteria, after discussions with the treatment team, it was decided to perform heating and test 5 the feasibility of heating also for this location by interstitial measurements.

During the first heating session, the water in the waterbolus was initially circulated at $25^{\circ} \mathrm{C}$ to reduce superficial heating. After 16 minutes, the patient patient complained of pain at the left eye, which was solved by a changed SAR setting. At the time of the $1^{\text {st }}$ treatment, the superficial interstitial thermometry points (2 and 16) were still assigned to be in tumour. As the temperature at these positions remained below $39.2^{\circ} \mathrm{C}$, we tried to raise temperatures by increasing the waterbolus temperature from 25 to $30^{\circ} \mathrm{C}$ after 38 minutes. The power was further increased to maximum $738 \mathrm{~W}$. 48 minutes after start of treatment the patient suddenly became panicky which made 

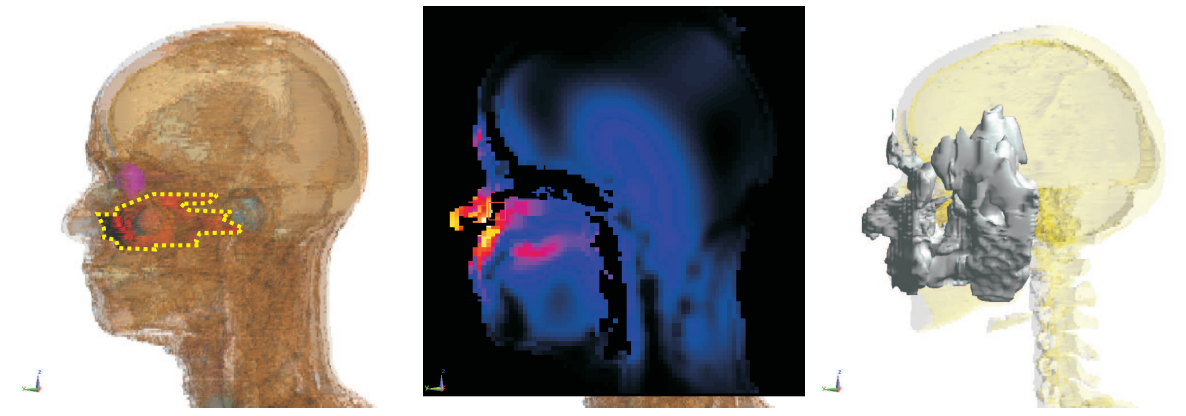

Figure 4. [Patient 2: Nasal cavity] Sagittal view of the anatomy (left), SAR distribution (middle) and 25\% iso-SAR volume in grey on top of the bony structures (right). The colorscale of the central SAR distribution is from black $(0 \%)$ to white $(100 \%)$.

us stop treatment. After the first treatment, with the aid of the specific thermometrylocalization CT, measurement points 2 and 16 were re-assigned to be in normal tissue. In the two subsequent heating sessions, that could be completed, we used a low waterbolus temperature $\left(\mathrm{T}_{a v} \sim 21^{\circ} \mathrm{C}\right)$. During these treatments the patient reported

5 hotspots at both eyes. We allocate these to be caused by local folds in the waterbolus that are not yet included in the model. However, no problems related to the eyes were reported in follow-up. Figure 7 shows the temperatures obtained during the three heating sessions. This figure shows also for patient 2 that, for all heating sessions, the average temperature measured in interstitial catheters was higher and more consistent than the avarage of the intraluminally measured temnperatures, i.e. within the mould.

\section{Patient 3: Thyroid}

The $3^{\text {rd }}$ patient was a 58 -year-old female with a recurrent follicular thyroid carcinoma. She was previously treated with surgery but refused a second surgery treatment, which would have included total laryngectomy. In stead, she was treated with IMRT $(16 \times 3.1 \mathrm{~Gy}, 4 / \mathrm{wk})$ and four times HT. Metal surgical clips $(\sim 5 \mathrm{~mm})$ located nearby the treatment field were identified in pre-treatment analysis as potentially treatment limiting.

Figure[5] shows the $25 \%$ iso-SAR volume as predicted by HTP. A TC of $94 \%$ was found and HTP further predicted no treatment limiting hotspots due to the metal implants. The patient agreed on interstitial thermometry for one treatment. Before the first treatment, two catheters were placed in the tumour which were removed afterwards. Because the tumour was diagnosed to extend up to the skin, we placed an additional waterbolus (six-antenna SHT bag (Van der Gaag et al. 2006)) between the patient's skin and the waterbolus of the HYPERcollar. By circulating the additional bolus at $40^{\circ} \mathrm{C}$, the skin underneath was isolated from cooling in order to obtain therapeutic temperatures in the whole tumour volume, i.e. up to the skin surface.

For the first treatment, the patient moved substantially and at the end of treatment positioning was substantially different as compared to planned positions $(3 \mathrm{~cm}$ in caudal direction). During treatment, the patient experienced heat at the front of the neck and at her cheeks. This had already been predicted by treatment planning but recalculations using the actual treatment position showed an even better agreement, 

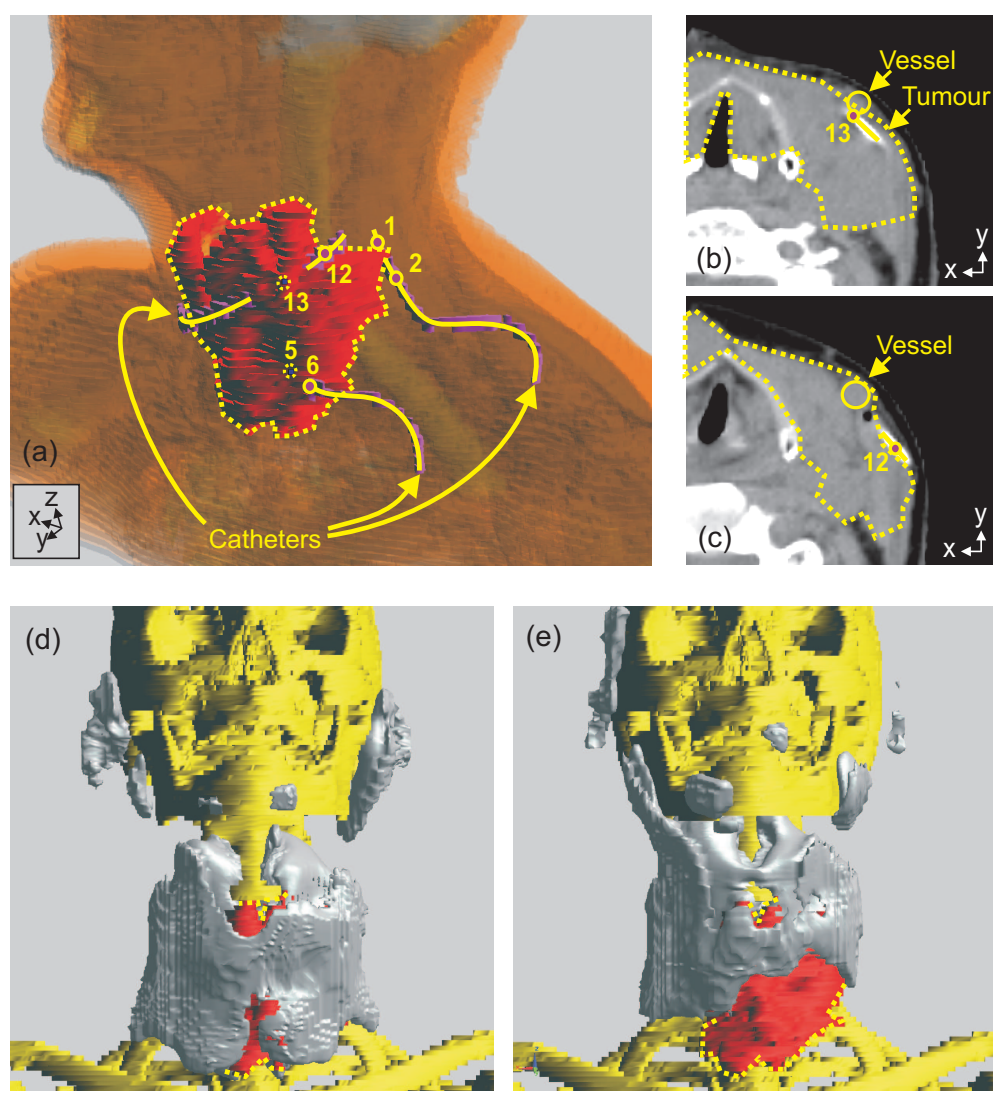

Figure 5. [Patient 3: Thyroid] Catheter locations (a-c) and 25\% iso-SAR volume in grey $(\mathrm{d}, \mathrm{e})$ for the patient in planned position $(\mathrm{d})$ and for the corrected actual patient positioning during treatment (e). The outline of the tumour is indicated by a yellow striped line and the patient model is shown either transparant (a) or only the tumour and bones (yellow) are shown (d,e). Projections of the catheters tracks are indicated in (a) by the yellow lines and the measurement locations are indicated by solid (outside the tumour), or dotted (inside the tumour), yellow circles.

as can be seen in the iso-SAR volume (Fig 5). Power was increased until $43^{\circ} \mathrm{C}$ was measured in the tumour (Figure 6). The temperature in point 13 was $3.2^{\circ} \mathrm{C}$ lower than the temperature measured by a sensor in the same probe that is $2 \mathrm{~cm}$ away (point 12). Analysis of the thermometry CT showed that this very large thermal gradient 5 was caused by major cooling due to a nearby large vessel (Figure 5).

Measurement point 5 revealed an unchanged temperature $\left(37.2^{\circ} \mathrm{C}\right)$. Inspection of Figure 5 shows that the unchanged temperature had been caused by the difference in positioning ("treatment" vs "planned" positions), i.e. this measurement point was located outside the area with high SAR due to the $3 \mathrm{~cm}$ shift. Also measurement point 6 was located outside this area but, due to the additional influence of the waterbolus that was circulated at an average temperature of $39.4^{\circ} \mathrm{C}$, this point still reached a maximum of $39.7^{\circ} \mathrm{C}$. Firstly, this case shows that it is possible to obtain temperatures that approximate $40^{\circ} \mathrm{C}$ when a large vessel is nearby, i.e. within mm's distance. Secondly, it shows that positioning and immobilization of the patient, especially in 

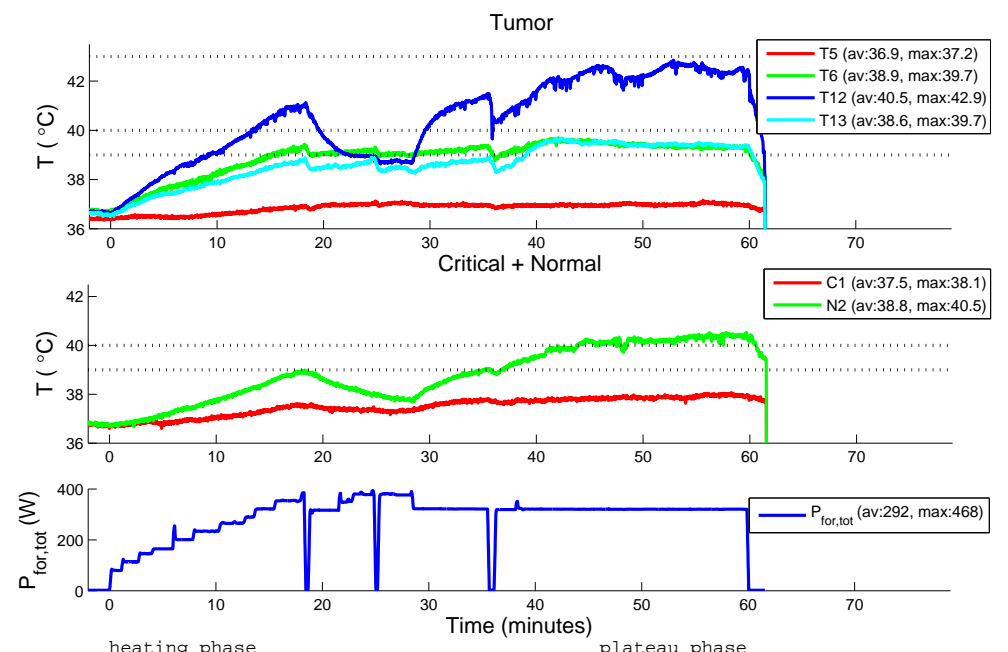

Figure 6. [Patient 3: Thyroid] Temperature and power versus time. This figure also graphically illustrates the "heating" and "plateau" phases. The legend shows the probe label and number (corresponding to Figure 5) and the average and maximum values of the respective curves in plateau phase. Temperature measurements for temperature-SAR analysis (see figure 8) were taken after 0,42 and 58 minutes

axial direction, is difficult but at the same time highly important. However, by reconstruction with HTP, we established that the inaccuracy of positioning in the axial direction results in an axial shift in heating area but no increased myelum heating, hence no additional risk of toxicity. Lastly, this case shows the high temperature 5 gradients that are present in $\mathrm{H} \& \mathrm{~N}$ HT, i.e. up to $1.6^{\circ} \mathrm{C} / \mathrm{cm}$, and hence the need for a highly focussed and accurately controlled treatment application.

\section{Verification of the treatment approach}

Figure 8 shows the correlation between temperature measurements and simulated SAR at the location of the probes. For example, for patient 3 , temperatures at 0,42 and 58 minutes were used since, at these times, the temperature and power profiles are (relatively) stable and no other change in treatment settings was indicated in the treatment report (see figure 6). Position, power and phase settings of the simulations were matched to the settings at the moment of the measurement. Figure 8 shows a fair to high correlation between measured temperatures and simulated powers for each patient separately. Hence, it preliminary confirms the rationale to use HTP guided SAR distribution optimization to increase tumour temperatures and heat to patients tolerance. This correlation is only moderate when combining the data of all three patients $\left(R^{2}=0.4\right)$, which is possibly due to large differences in perfusion and waterbolus cooling for different sites and different patients. This figure also indicates a better correlation for patient 2 (6th patient) and 3 (7th patient) with respect to patient 1 (2nd patient). This may be explained by a learning curve, but also by the fact that patient 1 had a tumour in the base of the tongue that is characterized by movements and changing volumes of air, decreasing the accuracy of HTP. 


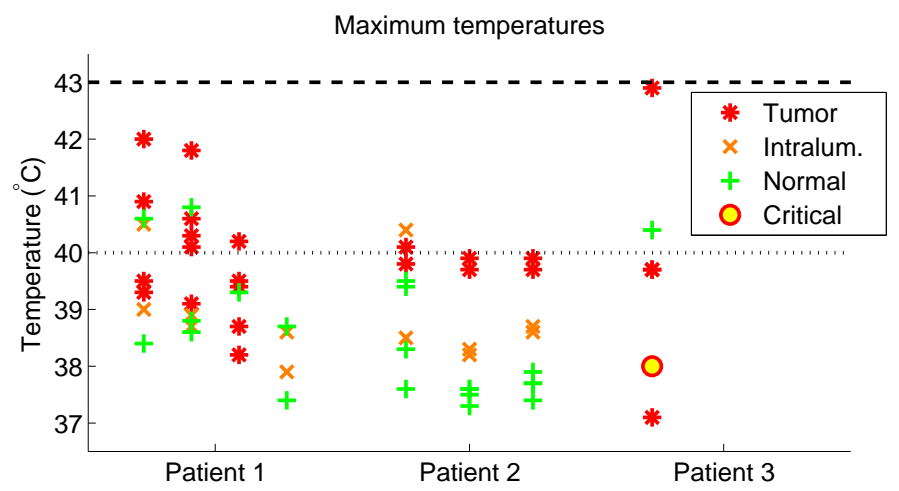

Figure 7. Maximum temperatures obtained during treatments for the three case patients. Maximum temperatures are presented because they illustrate better the potential of the treatment and are less influenced by confounding factors. Note that for patient $3,39.7^{\circ} \mathrm{C}$ was measured at two separate measurement points (presented as a single point in the graph). Further note that the low temperature points for patient 3 illustrate the need of accurate positioning, i.e. $37^{\circ} \mathrm{C}$ was measured outside the heating field, and the extremely inhomogeneous cooling in the $\mathrm{H} \& \mathrm{~N}$, i.e. $39.7^{\circ} \mathrm{C}$ was measured in the vicinity of a large vessel.

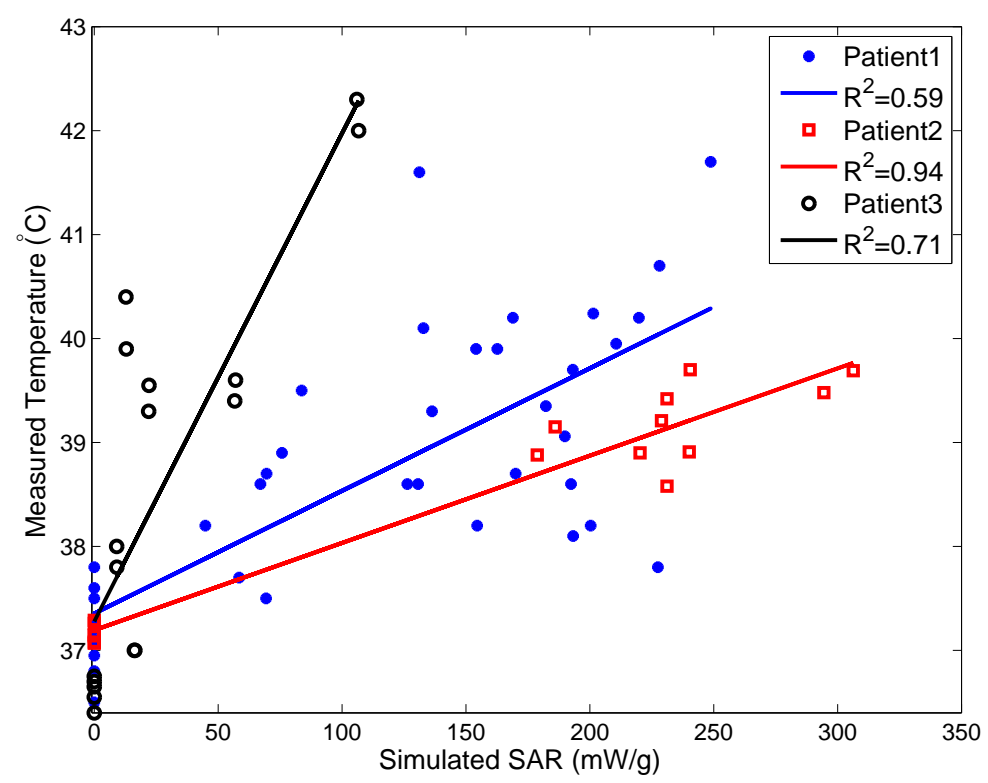

Figure 8. Temperature measurements, for patient 1 ("oropharynx"), patient 2 ("nasopharynx/nasal cavity") and patient 3 ("thyroid"), as function of local SAR, simulated using the clinically measured power and phase settings. Linear fits and corresponding $R^{2}$ values are shown for the three patients separately because the correlation of the combined data of the three patients was moderate, i.e. $R^{2}=0.40$. This figure shows a difference in slope of the linear fits between patients but a fair correlation on a per patient basis. 


\section{Discussion}

The head and neck region is characterized by many tissue transitions, large cooling vessels and the presence of critical tissue very nearby. Applying a good HT treatment to this region is therefore a challenging task. To tackle these challenges, we developed

5 a site-specific HT applicator for HT treatments in this complicated region. In this publication, we thoroughly analysed the treatments of three patient using the applicator design. Measured temperatures indicate the potential to induce effective $\mathrm{HT}$ in this region using the current standards in treatment application. However, the cases show, that application of HT in the H\&N region involves many aspects with complex interaction. Two main aspects will be treated separately below. Firstly, for this new area of deep HT application there is a high potential to enhance reliability of translating HTP settings into the clinic. Further, we will discuss the need of additional work on temperature measurements, or other means of on-line assessment of treatment quality, in order to assess and optimize the heating profiles. In general, we observe that a learning curve exists due to the novel challenges faced in this treatment application. Taking into account new evidence on dose effect relationships and our knowhow, we have deliberately set the standards of treatment quality high since we are convinced that this will convert into better clinical outcome. Higher standards require more thourough analyses but, fortunately, developments in simulation technology have provided the important tools needed to do so.

Translation of HTP settings A major item in an accurate translation of HTP settings into the clinic is the controllable and reproducible positioning of the patient in treatment position (Gellermann et al. 2000). In the current design this item has received limited attention and, at present, positioning is cumbersome and especially in the axial (z) direction not sufficiently accurate, i.e. around $\pm 1 \mathrm{~cm}$. Canters et al. (Canters et al. 2009) have shown that positioning accuracies for DHT have to be within $\pm 2.5 \mathrm{~cm}$ for a hotspot to target SAR ratio deviation of maximum $10 \%$. Using the five-fold difference in frequencies, this required accuracy for DHT can be converted to the necessity of $\pm 5 \mathrm{~mm}$ accuracy in positioning for H\&N HT at $433 \mathrm{MHz}$.

Since the SAR focus to target volume ratio is much smaller for the H\&N treatment, the sensitivity of heating quality to positioning inaccuracies is probably greater and, hence, an accuracy in the order of mm's is most probably required. Further, improvement of the positioning process also deserves attention because the level of comfort and required effort during positioning determines the stress of the treatment and the 35 personnel required.

Translation of HTP settings into the clinic is also dependent on the accuracy of the waterbolus model (Hornsleth 1996). For accurate SAR predictions we found that, since the waterbolus is flexible for conforming to the patients' skin, it's shape is clinically difficult to prescribe. Hence, we are planning investigations to assess the variability ${ }_{40}$ and related uncertainties in SAR to decide whether an optimized design is required. It should be noted that such a design is always a compromise between comfort and accuracy. The temperature distribution is - besides the power absorption profile - also heavily dependent on the cooling mechanisms in tissue and by the waterbolus, which has already been shown for e.g. superficial HT (Van der Gaag et al. 2006, Arunachalam et al. 2008, Juang et al. 2006). The only data to assess the cooling profile is from measurements at the in- and outflow of the applicator and at the skin surface. This uncertainty is even greater when applying an additional waterbolus to locally reduce 
surface cooling. Again we intend to use theoretical parameter studies to deduce guidelines for optimum use, as in Van der Gaag et al. (Van der Gaag et al. 2006). We anticipate that thorough parameter studies will provide 1) a better understanding of all aspects effecting the SAR and thermal distribution and 2) future directions for 5 improvements on the applicator system.

Dosimetry An important issue for a high quality treatment is knowledge of the applied dose, i.e. "dosimetry". For a hyperthermia treatment, a predictive dose remains difficult to define. The process of finding the treatment determining parameters may well be boosted if 3D thermal data becomes available. Therefore, assessment of the 3D temperature distribution during treatment is one of the biggest challenges that remains to be solved. Up to now, most hyperthermia centers rely on a limited amount of temperature measurements within intraluminal or interstitial catheters. While interstitial measurement points provide the most reliable source of temperature information, our early experience in the $\mathrm{H} \& \mathrm{~N}$ regions shows that placement of the catheters are also the most important cause of treatment related toxicity, in line with (van der Zee et al. 1998). Further, placing and documenting interstitial catheter tracks is complicated and specifically placed interstitial catheters often cannot remain in position for more than a week. Contrary, intraluminal measurements can be performed without toxicity and for deep hyperthermia a correlation with interstitial temperatures has been shown (Wust et al. 1998, Fatehi, van der Zee, Notenboom \& van Rhoon 2007). However, for the H\&N HT treatment, little agreement can be expected between interstitial and intraluminal temperatures since we observed that swallowing and breathing severely influences measurement results. Hence, a major improvement in $\mathrm{H} \& \mathrm{~N}$ specific thermometry technology 25 is warranted. Probably the best way to obtain temperature data for HT is by using non-invasive thermometry techniques (NIT) (Van Rhoon \& Wust 2006). Within the HT community, extensive research is directed towards measuring the 3D thermal distribution by MRI techniques (Gellermann et al. 2005, Gellermann et al. 2008, Stakhursky et al. 2009, Cheng et al. 2007). This technique is promising so but requires the availability of high-cost MRI equipment. Further, it is hampered by motion artifacts (Rieke \& Butts Pauly 2008, Gellermann et al. 2005) that can be pronounced in the $H \& N$ region due to e.g. swallowing and changes in bloodflow through large vessels. Also other techniques exist, e.g. microwave imaging and ultrasound, but clinical implementation of these is pending (Van Rhoon \& Wust 2006).

35 Alternative to improving measurement techniques is to further improve treatment planning and use patient-specific thermal models for predicting the temperature in the patient. In this paper, SAR based HTP already provided a welcome addition to the sparse thermometry points for the three cases described. The moderate correlation for all data combined indicates that no specific quantitative SAR threshold will predict for temperatures. However, correlation between predicted SAR and measured temperatures suggests that qualitative SAR parameters can be predictive for temperatures in the clinic. However, this correlation was only obtained by posttreatment simulations using settings measured in the clinic. Therefore a major effort in improving the translation of the model into the clinic would, besides a better treatment 45 quality and robustness, also lead to the possibility to upgrade thermometry at sparse thermometry points into knowledge about the 3D SAR and thermal distributions. 


\section{Conclusions}

In this paper, we present the first clinical data worldwide on deep heating in the head and neck using a phased array applicator. Three patient cases were analysed with a focus on the technology involved. The cases show the feasibility of hyperthermia 5 treatment of deep located tumors in the $\mathrm{H} \& \mathrm{~N}$ region by using the newly developed HYPERcollar. Measured temperatures, in this very challenging region, approach those reported for deep HT in the pelvic region, i.e. in some cases up to $43^{\circ} \mathrm{C}$. The current HYPERcollar is potentially an excellent option for deep heating in the H\&N, though still a gap still exists between the maximum and mean measured temperatures.

10 Partially, this gap is caused by the difficulty of arranging the setup of patient and applicator to match simulation settings. This has been proved by re-simulations with the true treatment configuration settings that significantly improved the correlation between simulations and measurements. We conclude that improving the control over - and accuracy of - patient positioning and waterbolus modeling are effective tools to enhance treatment quality, robustness and control. Especially, as the data show that improved SAR control is an effective means to arrive at higher temperatures.

This initial verification study forms the gateway to a more elaborate clinical validation of the efficiency of deep heating. This is a crucial step towards accurate control of the treatment which, combined with novel dynamic scanning techniques, makes focussed heating in the H\&N possible. We are convinced that precise heating will prove to be a very efficient tool in a multi-modality approach to improve clinical outcome combined with reduced toxicity.

\section{Acknowledgements}

This work was financially supported by the Dutch Cancer Society, grant: EMCR20094270. The authors further would like to thank the remaining technicians, physicians and physicists whom assisted during head and neck hyperthermia treatments: L. Groenendijk, G. Van der Wende - Bijdevaate, L. Verloop, A. Ameziane, H. Hakkenberg and M. Franckena for their help and useful hints. Lastly, B. Van Toorn and the other members of the Instrument Maintenance group are acknowledged for their manufacturing and maintenance work.

\section{References}

Amichetti M, Romano M, Busana L, Bolner A, Fellin G, Pani G, Tomio L \& Valdagni R 1997 Radiother Oncol 45, 155-158.

Arunachalam K, Maccarini P F \& Stauffer P R 2008 IEEE Trans Biomed Eng 55, 2397-2406.

Bakker J, Paulides M, Westra A, Schippers H \& Van Rhoon G n.d. Int J Hyperthermia . accepted for publication.

Bourhis J, Overgaard J, Audry H, Ang K, Saunders M, Bernier J, Horiot J, Le Matre A, Pajak T, Poulsen M, O'Sullivan B, Dobrowsky W, Hliniak A, Skladowski K, Hay J, Pinto L, Fallai C, Fu K, Sylvester R \& Pignon J 2006 Lancet 368, 843-854.

40 Canters R, Franckena M, Paulides M \& Van Rhoon G 2009 Phys Med Biol 54, 3923-3936.

Canters R, Franckena M, Van der Zee J \& Van Rhoon G 2008 Phys Med Biol 53, 6799-6820.

Cheng K, Stakhursky V, Stauffer P, Dewhirst M \& Das S 2007 Int J Hyperthermia. 23, 539-554.

Conley B 2006 J Clin Oncol 24, 1023-1024. Editorial.

Cummings B, Keane T, Pintilie M, Warde P, Waldron J, Payne D, Liu F, Bissett R, McLean M, Gullane P \& O'Sullivan B 2007 Radiotherapy and Oncology 85, 7-16.

El-Sayed S \& Nelson N 1996 J Clin Oncol 14, 838-847. 
Fatehi D, van der Zee J, de Bruijne M, Franckena M \& van Rhoon G C 2007 Int J Hyperthermia 23, 623-643.

Fatehi D, van der Zee J, Notenboom A \& van Rhoon G 2007 Strahlenther Onkol 183, 479-486.

Franckena M, Fatehi D, Bruijne M, Canters R, Norden Y, Mens J, Rhoon G \& Zee J 2009 Eur J Cancer 45, 1969-1978.

Gellermann J, Faehling H, Mielec M, Cho C, Budach V \& Wust P 2008 Int J Hyperthermia 24, 327335.

Gellermann J, Wlodarczyk W, Feussner A, Faehling H, Nadobny J, Hildebrandt B, Felix R \& Wust P 2005 Int $J$ Hyperthermia 21, 497-513.

Gellermann J, Wust P, Stalling D, Seebass M, Nadobny J, Beck R, Hege H, Deufhard P \& Felix R 2000 Int $J$ Radiat Oncol Biol Phys. 47, 1145-1156.

Hornsleth S 1996 Radiofrequency regional hyperthermia PhD thesis Aalborg University, Denmark.

Jellema A 2007 Radiation induced xerostomia in head and neck cancer patiens PhD thesis Vrije Universiteit Amsterdam.

15 Jensen J, Lambertsen K \& Grau C 2007 Radiotherapy and Oncology 85, 74-82.

Juang T, Stauffer P R, Neuman D G \& Schlorff J L 2006 Int J Hyperthermia 22, 527-544.

Kampinga H 2006 Int J Hyperthermia 22, 191-196.

Kohler T, Maass P, Wust P \& Seebass M 2001 Phys Med Biol 46, 2503-2514.

Kolkman-Deurloo I 2007 Intraoperative HDR Brachytherapy PhD thesis Erasmus Universiteit Rotterdam.

Levendag P, Peter R, Meeuwis C, Visch L, Sipkema D, de Pan C \& Schmitz P 1997 Radiotherapy and Oncology 45, 95-98.

Levendag P, Teguh D, Voet P, van der Est H, Noever I, de Kruijf W, Kolkman-Deurloo I, Prevost J, Poll J, Schmitz P \& Heijmen B 2007 Radiother Oncol 85, 64-73.

Neufeld E 2008 High Resolution Hyperthermia Treatment Planning PhD thesis ETH Zurich. Nr. 17947.

Paulides M, Bakker J, Neufeld E, Van der Zee J, Jansen P, Levendag P \& Van Rhoon G 2007 Int J Rad Oncol Biol Phys 68, 612-620.

Paulides M, Bakker J \& Van Rhoon G 2007 Int J Rad Oncol Biol Phys 68, 612-620.

30 Paulides M, Bakker J, Zwamborn A \& Van Rhoon G 2007 Int J Hyperthermia 23, 59 - 67.

Paulides M, Vossen S, Zwamborn A \& Van Rhoon G 2005 Int Rad Onc Biol Phys 63, 634-642.

Pignon J, Bourhis J \& Domenge, C D L 2000 Lancet 355, 949-955.

Rieke V \& Butts Pauly K 2008 J Mag Res Imag 27, 376-390.

Stakhursky V, Arabe O, Cheng K, Macfall J, Maccarini P, Craciunescu O, Dewhirst M, Stauffer P \& Das S 2009 Phys Med Biol 54, 2131-2145.

Sugahara T, van der Zee J, Kampinga H H, Vujaskovic Z, Kondo M, Ohnishi T, Li G, Park H J, Leeper D B, Ostapenko V, Repasky E A, Watanabe M \& Song C W 2008 Int J Hyperthermia 24, 123-40.

Terhaard C $20085^{\text {th }}$ edn Wolters Kluwer chapter 40, pp. 874-890.

40 Van der Gaag M, De Bruijne M, Samaras T, Van der Zee J \& Van Rhoon G 2006 Int J Hyperthermia 22, 637-656.

van der Zee J, Peer-Valstar J, Rietveld P, de Graaf-Strukowska L \& van Rhoon G 1998 Int J Radiat Oncol Biol Phys 40, 1205-1212.

van der Zee J, Vujaskovic Z, Kondo M \& Sugahara T 2008 Int J Hyperthermia 24, 111-22.

45 Van Rhoon G \& Wust P 2006 Int J Hyperthermia 21, 489-495.

Wust P, Gellermann J, Harder C, Tilly W, Rau B, Dinges S, Schlag P, Budach V \& Felix R 1998 Int J Radiat Oncol Biol Phys 41, 1129-1137. 